\title{
Detection efficiencies in high magnification events
}

\author{
Virginie Batista \\ PhD Student - IAP (Paris) \\ 98Bis Boulevard Arago \\ 75014 PARIS \\ FRANCE \\ E-mail: batistaliap.fr
}

\begin{abstract}
Since its pilot season in 1995, the PLANET (Probing Lensing Anomalies NETwork) collaboration has been active in nearly-continuous monitoring galactic microlensing events in order to detect extrasolar planets around the lens stars and to measure their abundance. For a number of observed events, it is however not clear whether a planet orbits the lens star, because the source trajectory might not probe any of the planetary caustics. Moreover, even in a favorable case, finite source effects might strongly affect and reduce a possible planetary signal. In such cases, one can compute the detection efficiency to planetary companion to the lens in order to estimate its probability.
\end{abstract}

We use the procedure underlined by Gaudi et al. 2002 to compute detection efficiencies of OGLE-2007-BLG-050, a single-lens-like microlensing event with showing strong finite source effects. In particular, we analyse the effect of the trajectory angle for a range of planet-star separation and mass ratio, which we use to caracterize the sensitivity of this microlensing event to planetary companions.

Please find the poster in attachment.

The Manchester Microlensing Conference: The 12th International Conference and ANGLES Microlensing Workshop

Manchester, UK

21-25 January, 2008 